\title{
A direct method for the identification of patterns of care using administrative databases: the case of breast cancer
}

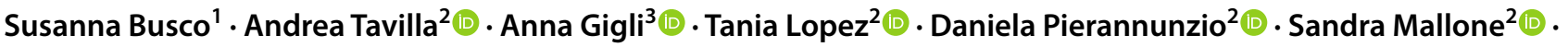

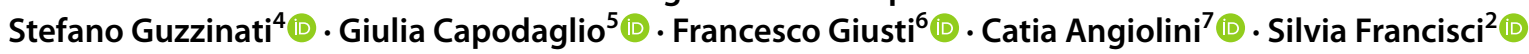

Received: 30 April 2020 / Accepted: 27 May 2021 / Published online: 26 July 2021

(c) The Author(s) 2021

\begin{abstract}
Objectives To identify and provide lists of procedures and drugs related to diagnosis and treatment of breast cancer. These lists can be used for the estimation of the cost of illness.

Methods The method consists of identifying lists of procedures/interventions/drugs related to the tumour of interest, drawn by a panel of expert clinicians and oncologists on the basis of clinical guidelines and current practice. The lists are applied to data referring to breast cancer female patients, collected by population-based Cancer Registries and linked at individual level with information on health care treatments. A comparison with lists obtained via the matched control method is implemented. Results The distribution of administered procedures and drug prescriptions is coherent with the patient clinical pathway: surgery is the main cause of hospitalization in the first year since diagnosis, diagnostic and monitoring interventions are more frequent in the following years (recurrences detection), and at end-of-life (palliative care). Most outpatient services are due to diagnosis and monitoring, one third of services in the first year since diagnosis is radiotherapy and chemotherapy. Drugs prescribed to patients and sold in pharmacy include hormonal drugs as first course treatment and analgesics as palliative care. Conclusions This direct method represents a valid alternative to the matched control method in describing patterns of care and costs related to the entire disease pathway. It is particularly suitable in case of cancer sites with complex patterns of care, such as breast cancer. The lists of codes developed here are based on international classification systems and can be easily applicable to other countries.
\end{abstract}

Anna Gigli

anna.gigli@irpps.cnr.it

1 UOC Programmazione Controllo E Governo Clinico-ASL Latina, Latina, Italy

2 National Centre for Disease Prevention and Health Promotion, National Institute of Health, Rome, Italy

3 Institute for Research on Population and Social Policies, National Research Council, via Palestro 32, 00185 Rome, Italy

4 Veneto Tumour Registry, Azienda Zero, Padua, Italy

5 Regional Epidemiology Service, Azienda Zero, Padua, Italy

6 Francesco Giusti, Brussels, Belgium

7 Breast Oncology Unit, Careggi University Hospital, Florence, Italy

\section{Introduction}

Administrative databases are more and more often used for estimating patterns of care and cost profiles of chronic diseases, such as cancer. Cost-of-illness studies are challenging because of the difficulties in identifying those costs that are due specifically to an illness.

There are two broad approaches used in cost-of-illness studies: the total cost approach, which consists of adding up all health expenditures referred to a cohort of patients or a selection of costs directly related to the disease of interest; and the matched control approach, which uses a comparison group of patients to isolate the incremental cost due to the disease of interest [1-3]. The matched control approach requires a proper matching between cases and controls, with respect to all confounding factors. This indirect approach is typically used in studies on the cost of cancer in the United States, based on SEER and Medicare data [4, 5], the latter containing information on cancer as well as non-cancer patients, and in New Zealand based on administrative health 
care datasets [6]. The total cost approach, when restricted to expenditures directly related to the disease of interest, requires an accurate and complete identification of these expenditures. This direct approach, also known as Attribution method [2], has been applied to identify and compare patterns of treatment of colorectal cancer patients during the first year since diagnosis between Italy and the United States [7].

The choice between these two approaches depends on the scope of the analysis, on the type of information available and on the illness of interest, as it will be illustrated in the discussion.

Aim of this paper is to identify lists of diagnostic and intervention procedures and drugs related to breast cancer using international classification systems. The resulting lists are obtained applying the direct method and are compared with those obtained using the indirect approach, in a selected subset of the study cohort for which information before cancer diagnosis is available.

The methodology is based on cancer registry data linked with administrative data and requires a strong involvement of expert clinicians and oncologists. Lists have been applied to estimate costs due to breast cancer diagnosis, treatment and follow-up in eight Italian regions, within the Epicost study [8]. Epicost represents the first attempt in Italy to provide population-based estimates of direct cancer costs across the patient pathway, when data at individual level are available.

\section{Materials and methods}

\section{Data sources}

The study includes data from population-based Cancer Registries (CRs) linked to four administrative sources that provide information on the following health care services: hospitalizations (Hospital Discharge database-HD), ambulatory services (Outpatient Services database-OPS), drugs prescribed to a patient and sold by a pharmacy (Drug Prescriptions database-DP), and high cost drugs administered to a patient in hospital (Hospital Pharmacy database-HP).

Information is collected at individual level and includes an anonymous personal identification code used for the record linkage with the CRs database, to trace all services provided to a single patient in the cancer registry in a given period of time. All personal data concerning individuals involved in this study were managed according to the current Italian privacy legislation [9] which identifies cancer registries as collectors of personal data for surveillance purposes without any need for explicit individual consent. The descriptive analysis of individual data did not involve any direct or indirect intervention on the population considered.
Population-based CRs collect data on all cancer diagnoses occurring in the population resident in the area covered by cancer registration. The following variables are included: date of birth, date of diagnosis, gender, vital status, topography and morphology code (according to ICDO-3 classification), diagnostic confirmation.

HD contains information on hospitalization. Each record refers to a single hospital episode and includes demographic variables (date of birth, sex, place of birth, place of residence), clinical variables (main diagnosis and up to five secondary diagnoses, main intervention/procedure and up to five secondary interventions/procedures coded according to ICD9-CM classification [10]), administrative variables (coded according to the DRG coding system, dates of admission and discharge). In this work, 24,480 records were analysed.

OPS contains information on outpatient services (for example diagnostic tests, ambulatory procedures). Each record refers to a single outpatient episode and includes the following variables: type of procedure and date of the episode. Procedures are coded according to a national coding system, based on ICD9-CM classification; 593,393 records were analysed.

DP and HP contain information on drugs and include the following variables: type of drug, coded according to the Anatomical Therapeutic Chemical (ATC) classification system [11] and date of prescription. Each record refers to a single drug; 313,719 records were analysed.

\section{Study cohort}

This study involves 8 Italian CRs, located in 8 out of 20 Italian regions, having at least 8 years of cancer registration: Veneto, Friuli Venezia Giulia, Milano in Northern Italy; Umbria, Firenze-Prato, Latina in Central Italy; Napoli, Palermo in Southern Italy. Regional coverage varies between 100\% (Friuli Venezia Giulia and Umbria regions) and 10\% (Latina in Lazio region); overall, they cover just over 10 million people, corresponding to about one sixth of the Italian population. A more detailed description of the study cohort can be found in [8].

The study design is cross-sectional: the study cohort includes patients alive at the most updated prevalence date available at the time of data collection, i.e., Jan 1st 2011, and having being diagnosed in a period of 8 years before (from Jan 1st 2003 to Dec 31st 2010). A total number of 49,270 prevalent women with breast cancer (ICD9-CM C50) are in the study cohort for the pool of CRs. Administrative data containing information on interventions, procedures and drugs is available for a 3-year interval, covering 2 years before to 1 year after prevalence date (from Jan 1st 2009 to Dec 31st 2011). 


\section{Methods}

We use a direct method to identify those procedures, interventions and drugs related to the tumour diagnosis, treatment and clinical follow-up. The method consists of identifying lists of events, i.e., procedures/interventions/ drugs, related to the tumour of interest, drawn by a panel of expert clinicians and oncologists on the basis of clinical guidelines [12]. The lists are associated to ICD9-CM codes for the identification of hospital and outpatient events and ATC codes for the identification of drugs. An event is cancer-related if the associated code belongs to the cancer-related list. In the case of hospitalization, a single episode may be associated with up to six diagnoses and six procedures/interventions, and a hospitalization is considered cancer-related if at least one code belongs to the cancer-related list.

The lists of cancer-related codes (D-lists) are reported in Online Appendix 1 separately for each type of service: hospitalization, outpatient, drugs. Each code is assigned to a clinically relevant category and a sub-category defined according to the type of the intervention as for hospitalizations and outpatient services or the type of treatment as for drugs.

We compare the results obtained using the direct method with those obtained by applying the matched control method to a subset of patient: according to this method, a comparison group of subjects without cancer diagnosis is matched with cases for all possible confounders and used to estimate the incremental access to health care services due to the breast cancer diagnosis. In our data set, since information on events is available for 2 years before prevalence date, for those patients in initial phase (i.e., diagnosed during year 2010) we also have information in a period prior to diagnosis, when we can assume that they undertook the same procedures as the general non-cancer population. Thus, the same patients in the initial phase are used as self-controls in the time interval spanning from 2 months up to 12 months before diagnosis [13]. Each patient belonging to this subset of the study cohort is linked to all events in the 10 months post(cases) and in the 10 months pre- (controls) cancer diagnosis. Notice that 2 months immediately prior to diagnosis were left out, to avoid possible biases due to cancer-related investigations in the pre-diagnostic period. The lists of cancer-related codes identified for hospitalizations, outpatient services and drugs by the matched control method (C-lists) are obtained in the following way: for each code, identifying a procedure/intervention/drug, cases and controls average occurrences are compared using a two-sample t-test for independent samples with unequal variances (Satterthwaite's Method) [14] (see Online Appendix 3).
Whenever the difference between cases and controls average occurrences is statistically significant $(p<0.05)$, the corresponding code is included. To measure the concordance between the $\mathrm{C}$-lists and the $\mathrm{D}$-lists we compute the ratio between the number of codes included in both $\mathrm{C}$-lists and D-lists and the number of codes included in the D-lists. Since frequencies of single codes can vary greatly, each code is weighted according to its number of occurrences. The cancer-related codes are then applied to the entire study cohort and distribution of occurrences are obtained for each phase of care, thus providing an overview of the patterns of care. To this purpose, we define three mutually exclusive phases of care: initial year following diagnosis (initial phase), last year of life (final phase), and the time between initial and final phase (continuing phase) and assign each case in the cohort to the phase of care she belongs on prevalence date, in the following way: if she had been diagnosed within 12 months before prevalence date and she is alive 12 months after prevalence date, she belongs to the initial phase; if she had been diagnosed more than 12 months before prevalence date and she is alive 12 months after prevalence date, she belongs to the continuing phase; if she died within 12 months after prevalence date, regardless of when she had been diagnosed, she belongs to the final phase. Women dying for causes other than cancer are considered as censored. It should be noticed that, although during her life span each woman can contribute to more than one phase of care, on prevalence date each patient belongs to only one phase of care, that is the phases of care are mutually exclusive. For each patient in the cohort we analyse information on events occurring in a 12-month period. This period spans from diagnosis onwards for patients in the initial phase, from death backward for patients in the final phase and from 6 months before to 6 months after the prevalence date for patients in the continuing phase.

\section{Results}

The direct method identifies 202 codes for hospitalization, 250 codes for outpatient procedures and 60 codes for pharmaceutical prescriptions. Codes are listed in Online Appendix 1. Of these, $99.6 \%$ codes for hospitalizations, $97.6 \%$ for outpatient services and $99.9 \%$ for drugs have been confirmed using the matched control method. The lists are applied to the study cohort, which is made of 7501 patients in the initial phase, 39,369 in the continuing phase and 2400 in the final one, corresponding to 15,85 and $5 \%$ of the cohort in initial, continuing and final phase, respectively.

Tables 1-3 describe the distribution by phase of care of the D-lists codes occurrences (grouped into categories and sub-categories), each table describes a different health care 


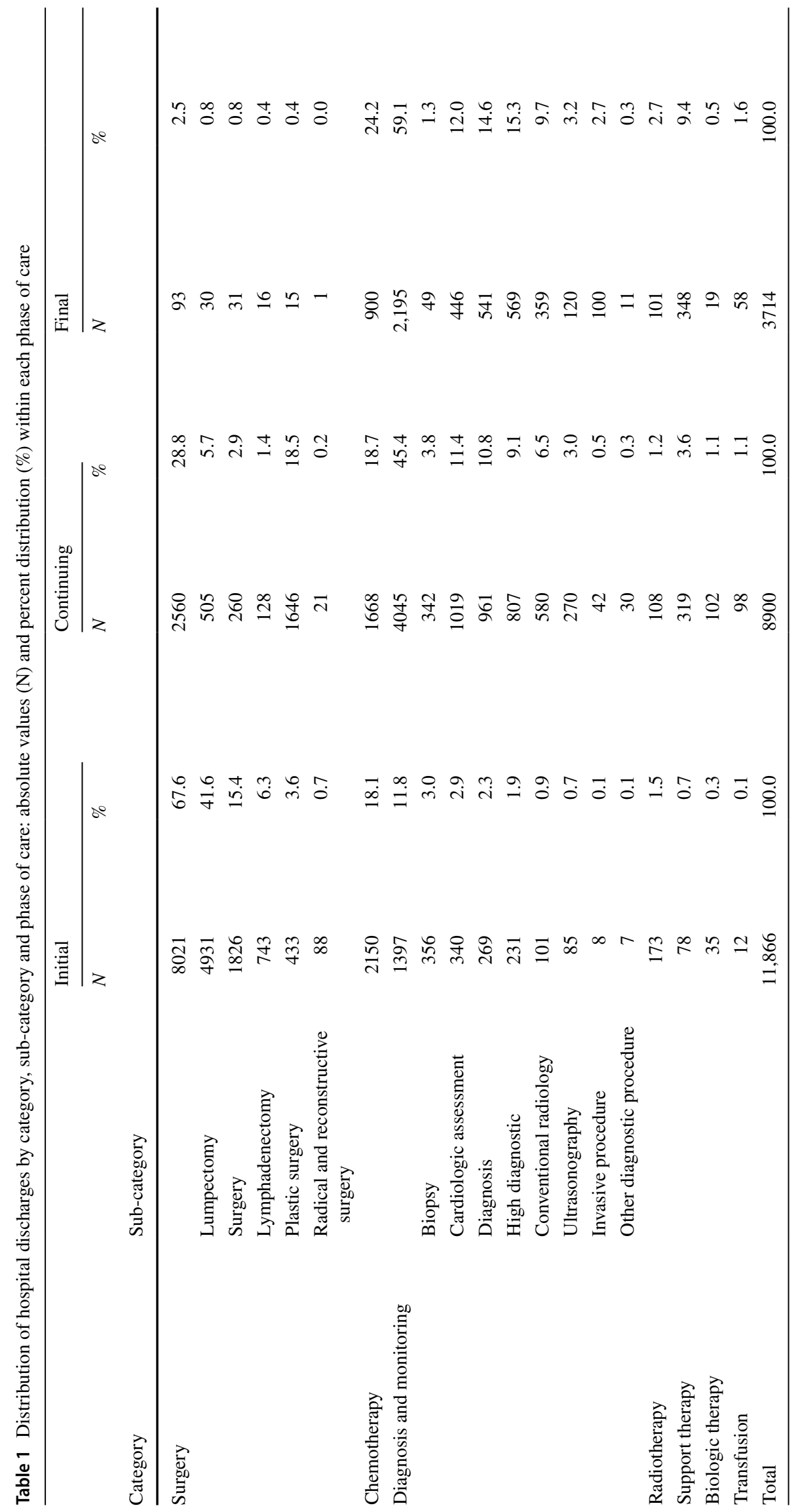




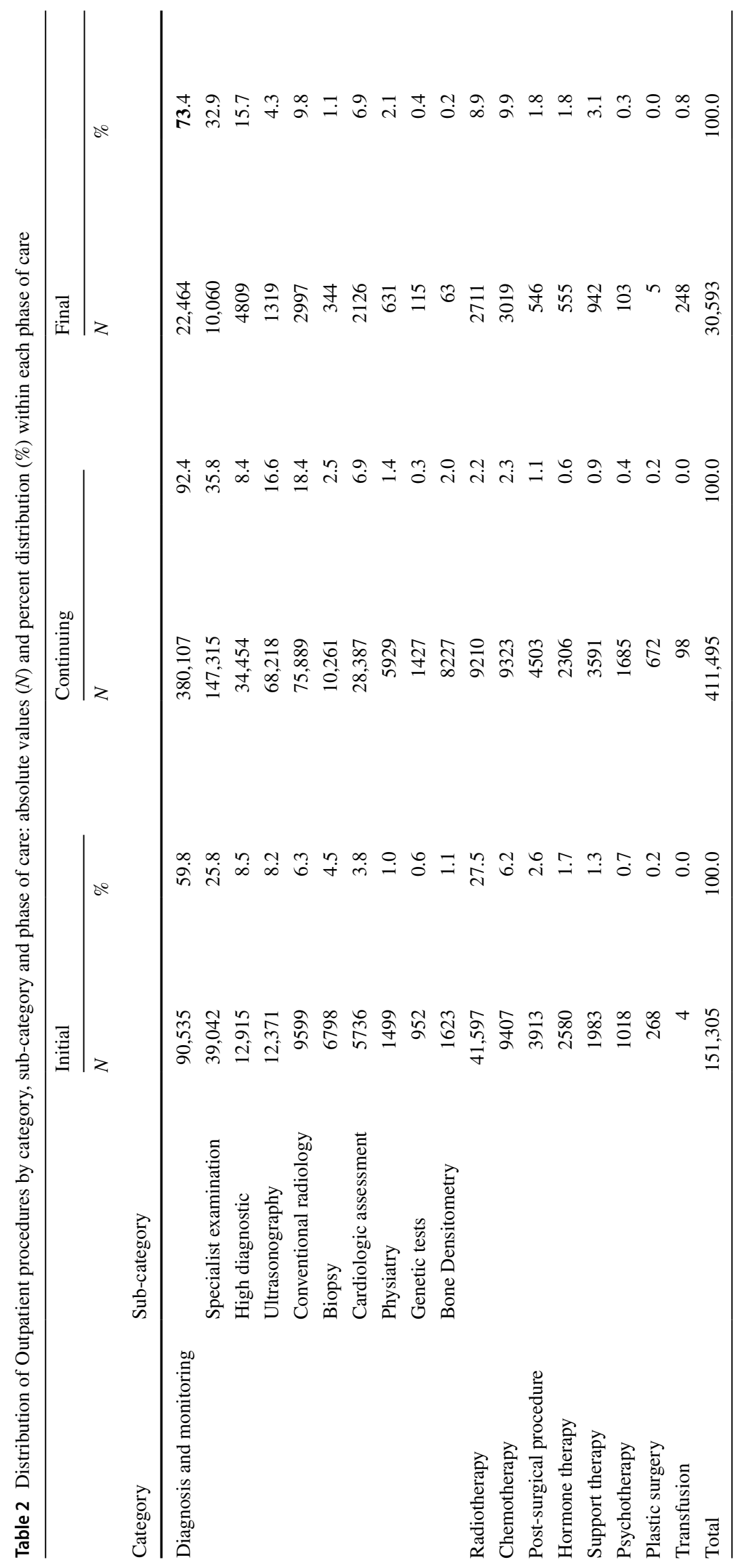


Table 3 Distribution of pharmacy drug prescriptions by category and phase of care: absolute values $(N)$ and percent distribution (\%) within each phase of care

\begin{tabular}{|c|c|c|c|c|c|c|}
\hline \multirow[b]{2}{*}{ Category } & \multicolumn{2}{|l|}{ Initial } & \multicolumn{2}{|c|}{ Continuing } & \multicolumn{2}{|l|}{ Final } \\
\hline & $N$ & $\%$ & $N$ & $\%$ & $N$ & $\%$ \\
\hline Hormone therapy & 18,947 & 63.5 & 93,373 & 67.5 & 2451 & 14.1 \\
\hline Cortisone & 4246 & 14.2 & 15,493 & 11.2 & 6684 & 38.3 \\
\hline Antiemetic & 2884 & 9.7 & 1579 & 1.1 & 844 & 4.8 \\
\hline Analgesic & 1568 & 5.3 & 12,4 & 9.0 & 6860 & 39.3 \\
\hline Myelopoietic growth factor & 1115 & 3.7 & 435 & 0.3 & 166 & 1.0 \\
\hline Bisphosphonate & 928 & 3.1 & 14,247 & 10.3 & 288 & 1.7 \\
\hline Chemotherapic drug & 136 & 0.5 & 722 & 0.5 & 94 & 0.5 \\
\hline Hematopoietic growth factors & 31 & 0.1 & 83 & 0.1 & 49 & 0.3 \\
\hline Total & 29,855 & 100 & 138 & 100 & 17 & 100 \\
\hline
\end{tabular}

service. Tables A2.1-A2.3 in Online Appendix 2 report, separately for each phase of care, the ten most frequently used codes in each D-list. Notice that at the time of the study information collected in the HP database, containing high cost drugs administered in hospital, such as some types of chemo- and immuno-therapeutic drugs, was incomplete and very variable among regions. Consequently, results regarding the HP database are merely qualitative: the ATC codes were subjected to validation and are listed in Online Appendix 1 , but no frequency table is shown.
Details of the HD database are illustrated in Table 1, where each hospitalization is classified according to the main cancer-related intervention/procedure. In 12 months, our cohort of patients experienced 24,480 hospitalizations, nearly half of which $(11,866)$ occurred during the initial phase of care, $36 \%$ (8900) in continuing phase and $15 \%$ (3714) in final phase.

In initial phase, surgery is the most frequent procedural category (with conservative surgery-lumpectomy-prevailing over the other surgeries), followed by chemotherapy and diagnosis and monitoring. In continuing phase

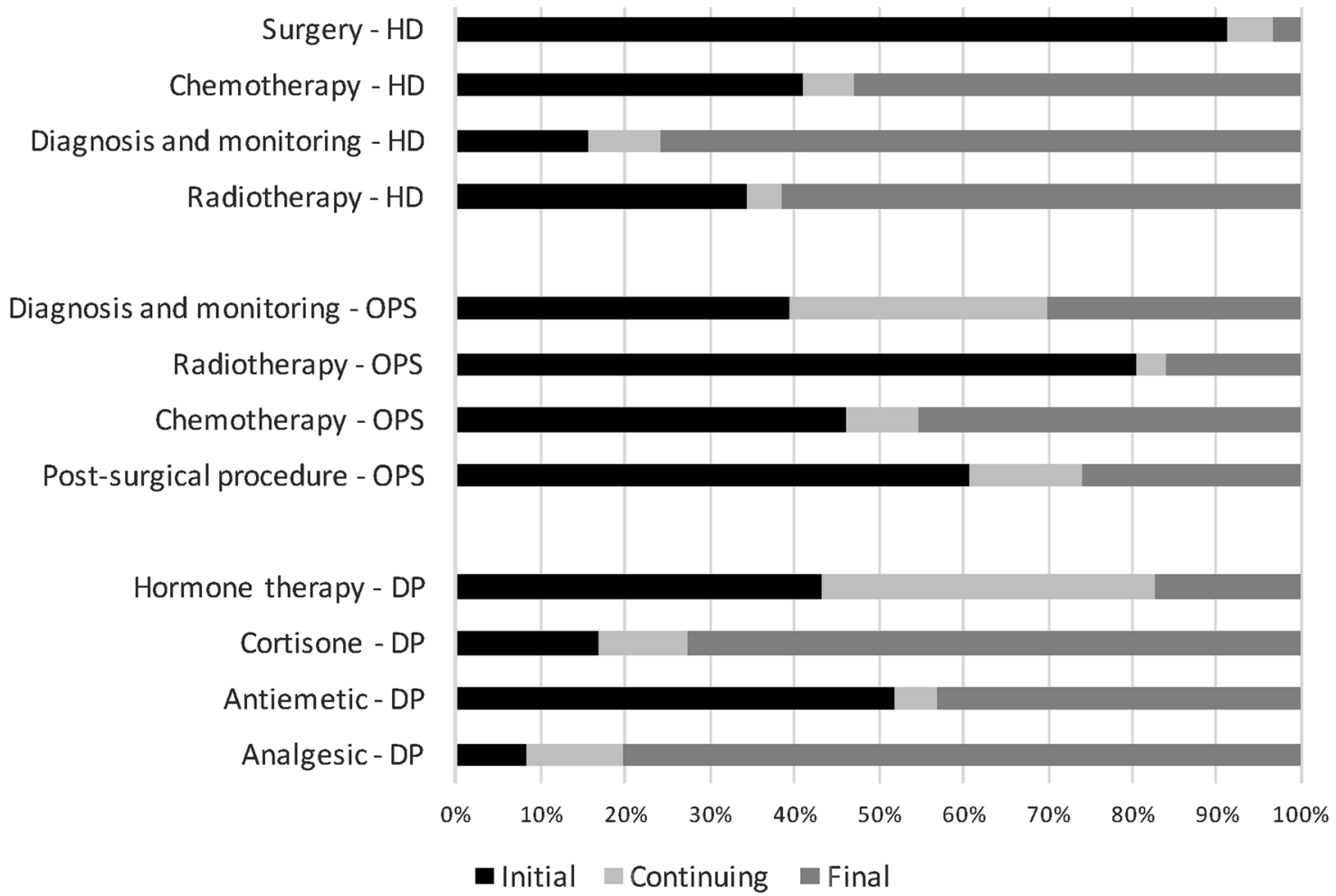

Fig. 1 Percent distribution by phases of care of four main categories in each data source: Hospital (HD), outpatient (OPS), pharmacy drugs (DP) 
diagnosis and monitoring category is the most frequent, followed by surgery (with plastic surgery prevailing) and chemotherapy. In final phase, diagnosis and monitoring is the most frequent category, followed by chemotherapy and support therapy. Within the diagnosis and monitoring category, the most frequent sub-category is biopsy in initial phase, cardiologic assessment in continuing phase and high diagnostic in final phase.

Table A2.1 describes the ten most frequent single cancerrelated procedures/interventions undertaken during hospitalization, percentages are computed over the total of cancerrelated procedures in each phase of care. In initial phase resection of quadrant of breast, chemotherapy and excision of axillary lymph node are the most frequent procedures accounting for about $35.5 \%$ of the total; in continuing phase chemotherapy represents the second most frequent procedure followed by diagnostic and monitoring procedures (Electrocardiogram, Routine chest X-rays); a similar pattern holds in final phase.

Details of the OPS database are reported in Table 2. Notice that standard blood tests (35 codes) and genetic markers ( 9 codes) although included in the D-list of Outpatient services are excluded from the frequency distribution of Table 2: they are individually prescribed (one test corresponds to one record) and account for a huge amount of records, so their inclusion in Table 2 would have biased the frequency distribution.

A total of 593,393 outpatient procedures were administered to our cohort of patients in 12 months: $25 \%(151,305)$ in initial phase, $70 \%(411,495)$ in continuing phase and 5\% $(30,593)$ in final phase. In initial phase, nearly $60 \%$ of procedures belong to the diagnosis and monitoring category (with specialist examination being the most frequent), followed by radiotherapy and chemotherapy. The same pattern is observed in the remaining phases, with varying percentages: $92.4 \%$ of diagnosis and monitoring procedures (specialist examination, radiology and ultrasonography) in continuing phase; $73.4 \%$ of diagnosis and monitoring procedures (specialist examination, high diagnostic, radiology being the more prevalent) in final phase.

Looking at the ten most frequent outpatient procedures by phase of care reported in Table A2.2: check-up examinations is in the first place in all three phases; initial and final phases of care have the same pattern (including chemotherapy, cardiologic assessment, high diagnostic) except for radiotherapy, which is present in initial phase only. In continuing phase, all procedures belong to the category diagnosis and monitoring (i.e., check-up examinations, conventional radiology, cardiologic evaluation, ultrasonography and DXA).

Table 3 describes the distribution of pharmacy drug prescriptions (i.e., drugs prescribed to a patient and sold by a pharmacy).
The most prescribed drugs in initial phase are hormone therapies, followed by cortisone-based drugs and anti-emetics, together representing $87 \%$ of drug prescriptions. In continuing phase, hormone therapy is followed by analgesics and cortisone-based drugs (together they represent $88 \%$ of total prescriptions). In final phase, analgesics are the most prescribed drugs, followed by cortisone-based drugs and hormone therapy (together representing $92 \%$ of prescriptions). Anti-emetics are present mainly in initial and final phases (9.7 and 4.8\%, respectively), and are most likely related to chemotherapy. Notice that chemotherapy drugs are poorly represented in this database, which describes only occurrences of drugs sold in a pharmacy. Instead, they can be found in HP database (high cost drugs administered to a patient in hospital), and as procedures in HD (hospital) and OPS (outpatient) databases.

Table A3.3 shows the lists of the ten most frequently drugs sold by pharmacies and reported in the DP database. In initial and continuing phases an anti-estrogen (Tamoxifen) is in the first place, followed by two aromatase inhibitors (Letrozole and Anastrozole). Two bi-phosphonate drugs used for bone diseases (Alendronic Acid and Risedronic Acid) are present in continuing phase only. In final phase, the most frequent drug is a cortisone-based drugs (Dexamethasone), followed by an analgesic (Fentanyl) and another cortisone-based drug (Prednisone).

Figure 1 illustrates the distribution by phase of care of the four most frequent (covering at least $90 \%$ of total occurrences) hospital and outpatient categories of procedures and pharmacy drug prescriptions; each category is weighted by the percent distribution of patients by phase of care.

Surgery is performed only in hospital setting and mainly during initial phase (91\%). Radiotherapy in hospital setting is mostly administered in final phase (62\%) while in outpatients setting is mostly administered in initial phase (80\%). Chemotherapy in hospital is delivered more frequently in final phase (53\%) than in initial phase (41\%), while in outpatient setting is equally distributed between initial and final phases. Diagnostic procedures performed in hospital are more frequent in final phase (76\%), while in outpatient setting are equally distributed between the three phases. Regarding pharmacy drug prescriptions, distribution patterns vary: hormone therapy is equally distributed between initial and continuing phase; antiemetic drugs are more frequently used in initial and final phase; cortisone-based drugs and analgesics are largely prescribed in final phase.

\section{Discussion}

This paper introduces a direct method consisting of lists of healthcare services related to diagnosis, treatment and follow-up of women with breast cancer. These lists are 
identified using clinical criteria and used for the estimation of the expenditures directly related to the cancer diagnosis.

This method has already been introduced in cost-ofillness studies, the novelty here is its application to three separated phases of the clinical pathway, from diagnosis to possible recovery or death.

The distribution by phase of care of the occurrences of the D-lists procedures and drug prescriptions is coherent with the patient clinical pathway: surgery is the main cause of hospitalization in the first year since diagnosis; diagnostic and monitoring interventions are more frequent in the following years (to detect possible recurrences), and at the end of life (to arrange palliative care); most ambulatory services belong to diagnosis and monitoring category; one third of services in the initial phase is radiotherapy and chemotherapy; drugs prescribed to patients and sold in pharmacy include hormone therapy in initial and continuing phase and analgesics in final phase.

The D-lists include specific codes related to breast cancer, as well as more generic ones, such as transfusion or support therapy or drugs classified as analgesics or antiemetics. This inclusive approach allows to reduce the risk of under-reporting cancer-related events, with the consequent under-estimation of medical expenditures indirectly associated with breast cancer [3].

The D-lists provided in this paper have been compared to the C-lists obtained by applying the matched control method that uses patients before the diagnosis of breast cancer as self-controls. There is over 97\% of concordance; the very few exceptions correspond to: (i) procedures and prescriptions that have not being administered to our cohort of patients, neither before nor after the cancer diagnosis; these codes are nevertheless included in the lists according to clinical criteria; (ii) generic procedures and prescriptions that occur before as well as after the cancer diagnosis (for example X-rays or cortisone); however, since our cohort is made of cancer patients, we assume that after diagnosis they were prescribed because of the cancer; (iii) cancer-related procedures and prescriptions that appear before cancer diagnosis, because for a few patients the period of pre-cancer investigation (which we excluded from the comparison) was longer than 2 months; notice that the choice of the 2-month cut off is appropriate for the large majority of cases. There are some limitations in this work.

The same lists of codes have been used for all phases of care. However, some less specific codes (for example Electrocardiogram or Routine chest X-rays) may be used for more than one chronic condition, and may not be completely suitable for elderly patients with other comorbidities, and in the continuing phase, which includes a mixture of patients with different clinical patterns: patients cured, patients with relapses and patients in chronic condition. A further improvement of the methodology would be to apply differential lists of codes according to clinical variables that possibly influence the patterns of care, such as phase of care, age and stage at diagnosis.

The D-lists are applied to cases diagnosed in the period Jan 1st 2003 to Dec 31st 2010 and refer to treatments administered in that period. Since then there has been a great improvement in drugs, especially immune-therapies based on chimeric monoclonal antibodies (such as Atezolizumab, Pembrolizumab or Pertuzumab), enzyme inhibitors (such as Abemaciclib, Olaparib, or Everolimus), proteinbound chemotherapies (such as Nab-paclitaxel), or other antineoplastic drugs (such as Eribulin). These drugs are not included in the present D-list and their inclusion is recommended in a study based on more recent data.

Finally, the development of the D-lists is quite time consuming and requires the engagement of a panel of experts. Notwithstanding, it should be emphasized that, based on the work produced in this study, the effort required for the revision of the classification system, for the update of the lists or for the adaptation of the lists to other cancer sites, would be minimal.

The approach presented has several strengths.

Firstly, the identification of cancer-related events allows us to describe their occurrences across phases of care, to identify specific cost drivers and, consequently, to suggest best strategies in a public health framework. For example, costs of a chemotherapy session provided in hospital setting is more than five-fold compared to outpatient setting.

Secondly, the direct approach does not require a comparison cohort (which in Italy is not available to CRs), thus avoiding possible biases in the results due to mismatches between cases and controls: indeed, a satisfying degree of matching might be difficult to obtain when dealing with chronic diseases with multiple risk factors that are common to other pathological conditions; moreover, information on risk factors are rarely included in administrative databases such as those used in the present study.

Thirdly, health services in Italy are administered on regional basis and health care databases are available in all regions, in a standardized format. The D-lists based on data from the eight regions included in the Epicost study can be easily applied to other Italian regions, thus producing standardized and highly comparable results. The only adjustment required is the integration of regional-specific codes used for outpatient services.

Finally, the lists of codes developed here are based on international classification systems and can be easily applicable to other countries. As an example, in the ongoing Innovative Partnership for Action Against Cancer (iPAAC) financed by the European Commission the methodology has been proposed for application to other European countries, such as Belgium, Spain, Norway and Poland [15]. 
Supplementary Information The online version contains supplementary material available at https://doi.org/10.1007/s10198-021-01327-8.

Acknowledgements We thank Adele Caldarella (Tuscany Cancer Registry), Mario Fusco (Napoli Cancer Registry), Silvia Iacovacci (Latina Cancer Registry), Walter Mazzucco (Palermo Cancer Registry), Massimo Rugge (Veneto Cancer Registry), Antonio Giampiero Russo (Milan Cancer Registry), Diego Serraino (Friuli Venezia Giulia Cancer Registry), Fabrizio Stracci (Umbria Cancer Registry) for collecting and providing data and correcting data after quality controls. Pharmacoepidemiologists Giuseppe Traversa and Roberto Da Cas and medical oncologist Manuela Tamburo De Bella contributed to the investigation on drug prescriptions.

Author contributions $\mathrm{SB}, \mathrm{CA}, \mathrm{AG}, \mathrm{SF}$ and $\mathrm{SG}$ drafted the study protocol and designed the study. SG, GC, SF, AG, SM, AT, DP, TL implemented the quality control checks. AT e DP did the statistical analysis. AT developed the SAS code, with the support of SG, GC and SF. SF, AG, TL, DP and AT supported SB in the interpretation and clinical implication of study results. AT, SF, AG, TL drafted the manuscript. All authors revised the preliminary results, reviewed and approved the final version of this manuscript.

Funding The study was funded by the Italian Ministry of Health (PROGRAMMA CCM 2014) and by the European Commission (Work Programme 2017, Grant Number 801520 HP-JA-2017 “Innovative Partnership for Action Against Cancer"). The funding bodies had no role in the study design, collection, analysis or interpretation of data, the writing of the report, or the decision to submit the article for publication.

Data availability Dataset supporting our findings is available, according to AIRTUM guidelines, at the following website: www.registritumori.it.

\section{Declarations}

Conflict of interest The authors declared that there is no conflict of interest.

Ethical approval The approval of a research ethic committee is not required, since this study is a descriptive analysis of individual data without any direct or indirect intervention on patients (Decreto del Presidente del Consiglio dei Ministri, 3/3/2017, Identificazione dei sistemi di sorveglianza e dei registri di mortalità, di tumori e di altre patologie, 17A03142, GU Serie Generale n.109 del 12-05-2017 (Available at: http://www.gazzettaufficiale.it/eli/id/2017/05/12/17A03142/sg, last access: 23/04/2020).

Consent to participate The Italian legislation identifies Cancer Registries as collectors of personal data for surveillance purposes without explicit individual consent.

Open Access This article is licensed under a Creative Commons Attribution 4.0 International License, which permits use, sharing, adaptation, distribution and reproduction in any medium or format, as long as you give appropriate credit to the original author(s) and the source, provide a link to the Creative Commons licence, and indicate if changes were made. The images or other third party material in this article are included in the article's Creative Commons licence, unless indicated otherwise in a credit line to the material. If material is not included in the article's Creative Commons licence and your intended use is not permitted by statutory regulation or exceeds the permitted use, you will need to obtain permission directly from the copyright holder. To view a copy of this licence, visit http://creativecommons.org/licenses/by/4.0/.

\section{References}

1. Akobundu, E., Ju, J., Blatt, L., Mullins, C.D.: Cost-of-illness studies: a review of current methods. Pharmacoeconomics 24, 869-890 (2006)

2. Caulley, L., Thavorn, K., Rudmik, L., Cameron, C., Kilty, S.J.: Direct costs of adult chronic rhinosinusitis by using 4 methods of estimation: results of the US Medical Expenditure Panel Survey. J. Allergy. Clin. Immunol. 136, 1517-1522 (2015)

3. Coughlan, D., Yeh, S.T., O'Neill, C., Frick, K.D.: Evaluating direct medical expenditures estimation methods of adults using the medical expenditure panel survey: an example focusing on head and neck cancer. Value. Health. 17, 90-97 (2014)

4. Mariotto, A.B., Yabroff, K.R., Shao, Y., Feuer, E.J., Brown, M.L.: Projections of the cost of cancer care in the United States: 2010 2020. J. Natl. Cancer Inst. 103, 117-128 (2011)

5. Banegas, M.P., Yabroff, K.R., O'Keeffe Rosetti, M.C., et al.: Medical care costs associated with cancer in integrated delivery systems. J. Natl. Compr. Canc. Netw. 16, 402-410 (2018)

6. Blakely, T., Atkinson, J., Kvizhinadze, G., Wilson, N., Davies, A., Clarke, P.: Patterns of cancer care costs in a country with detailed individual data. Med. Care. 53, 302 (2015)

7. Gigli, A., Warren, J.L., Yabroff, K.R., Francisci, S., Stedman, M., Guzzinati, S., Giusti, F., Miccinesi, G., Crocetti, E., Angiolini, C., Mariotto, A.: Initial treatment for newly diagnosed elderly colorectal cancer patients: patterns of care in Italy and the United States. J. Natl. Cancer Inst. Monogr. 2013, 88-98 (2013)

8. Francisci, F., Guzzinati, S., Capodaglio, G., et al.: Patterns of care and cost profiles of women with breast cancer in Italy: EPICOST study based on real world data. Eur. J. Health. Econ. 21, 1003-1013 (2020). https://doi.org/10.1007/s10198-020-01190

9. Decreto del Presidente del Consiglio dei Ministri. Identificazione dei sistemi di sorveglianza e dei registri di mortalita', di tumori e di altre patologie. GU Serie Generale n.109 (2017)

10. World Health Organization: International classification of diseases 9th revision, clinical modification. World Health Organization, Geneva (1997)

11. WHO Collaborating Centre for Drug Statistics Methodology: ATC classification index with DDDs, 2019. Oslo, Norway (2018)

12. Linee guida AIOM 2010: Neoplasie della mammella. Milano, Italy (2010)

13. Sheehan, D.F., Criss, S.D., Chen, Y., Eckel, A., Palazzo, L., Tramontano, A.C., et al.: Lung cancer costs by treatment strategy and phase of care among patients enrolled in Medicare. Cancer. Med. 8, 94-103 (2019)

14. Satterthwaite, F.E.: An approximate distribution of estimates of variance components. Biometrics. Bull. 2, 110-114 (1946)

15. The iPAAC project. https://www.ipaac.eu. Accessed Apr 23, 2020.

Publisher's Note Springer Nature remains neutral with regard to jurisdictional claims in published maps and institutional affiliations. 